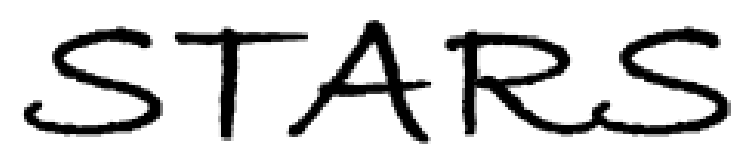

University of Central Florida

STARS

Faculty Bibliography 2000s

Faculty Bibliography

$1-1-2009$

\title{
Parker problem in Hall magnetohydrodynamics
}

Bhimsen K. Shivamoggi

University of Central Florida

Find similar works at: https://stars.library.ucf.edu/facultybib2000

University of Central Florida Libraries http://library.ucf.edu

This Article is brought to you for free and open access by the Faculty Bibliography at STARS. It has been accepted for inclusion in Faculty Bibliography 2000s by an authorized administrator of STARS. For more information, please contactSTARS@ucf.edu.

\section{Recommended Citation}

Shivamoggi, Bhimsen K., "Parker problem in Hall magnetohydrodynamics" (2009). Faculty Bibliography 2000s. 2135.

https://stars.library.ucf.edu/facultybib2000/2135

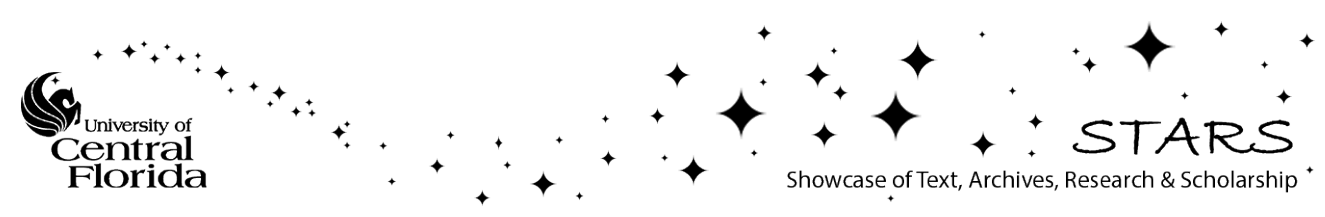




\section{Parker problem in Hall magnetohydrodynamics}

Cite as: Phys. Plasmas 16, 052111 (2009); https://doi.org/10.1063/1.3140055

Submitted: 02 September 2008. Accepted: 28 April 2009. Published Online: 28 May 2009

Bhimsen K. Shivamoggi

\section{ARTICLES YOU MAY BE INTERESTED IN}

Steady and unsteady Hall magnetohydrodynamics near an X-type magnetic neutral line Physics of Plasmas 18, 052304 (2011); https://doi.org/10.1063/1.3581092

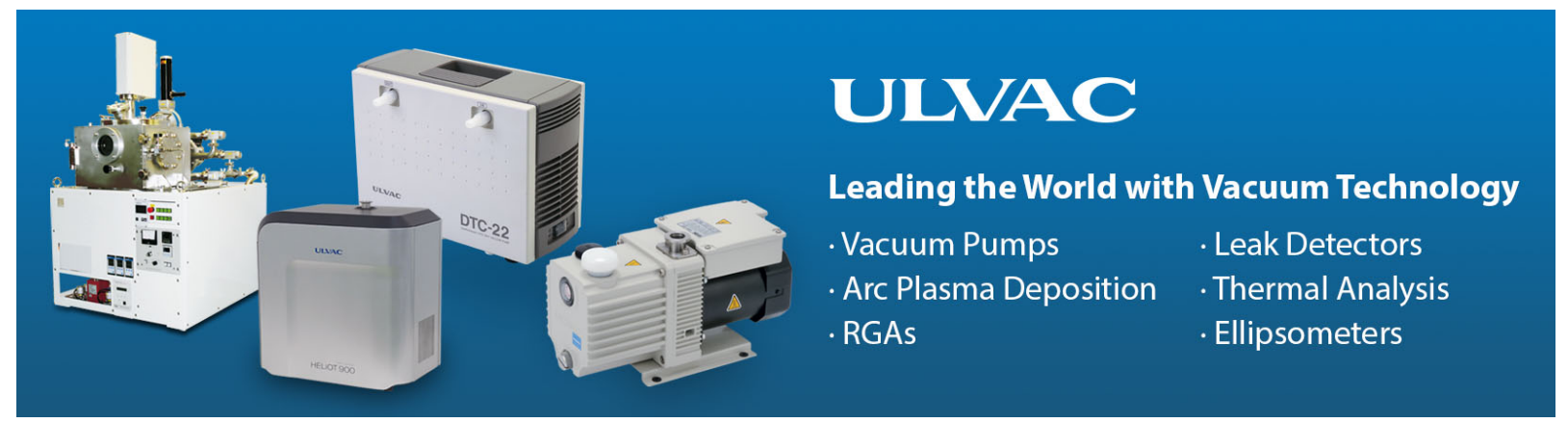




\section{Parker problem in Hall magnetohydrodynamics}

Bhimsen K. Shivamoggi ${ }^{\text {a) }}$

Los Alamos National Laboratory, Los Alamos, New Mexico 87545, USA

(Received 2 September 2008; accepted 28 April 2009; published online 28 May 2009)

The Parker problem in Hall magnetohydrodynamics (MHD) is considered. Poloidal shear superposed on the toroidal ion flow associated with the Hall effect is incorporated. This is found to lead to a triple deck structure for the Parker problem in Hall MHD, with the magnetic field falling off in the intermediate Hall-resistive region more steeply (like $\left.1 / x^{3}\right)$ than that (like $1 / x$ ) in the outer ideal MHD region. (C) 2009 American Institute of Physics. [DOI: 10.1063/1.3140055]

\section{INTRODUCTION}

Numerical simulations ${ }^{1}$ suggested that magnetic reconnection in resistive magnetohydrodynamics (MHD) can be driven by a magnetic flux pile-up. In this process, magnetic field builds up upstream of a Sweet ${ }^{2}-$ Parker $^{3}$ current sheet (this field buildup strengthens as the resistivity is decreased), which leads to an increase in the outflow downstream of the current sheet. This provides for a remedy for the SweetParker "bottleneck" limiting the outflow and enabling reconnection to proceed at the externally imposed rate. However, as the resistivity is decreased further, the development of large magnetic pressure gradients upstream of the current sheet opposes the ion inflow, ${ }^{5}$ which is the only means in resistive MHD to transport magnetic flux into the reconnection layer. The magnetic flux transport into the reconnection layer (and hence the reconnection rate) is reduced-the socalled pressure problem. ${ }^{6}$ The Hall effect ${ }^{7}$ can overcome the pressure problem ${ }^{8,9}$ thanks to the decoupling of electrons from ions on length scales below the ion skin depth $d_{i}$. So, if the reconnection-layer width is less than $d_{i}$, the electron inflow can keep on going, which transports the magnetic flux into the reconnection layer and hence reduces the flux pileup. Dorelli ${ }^{10}$ considered the role of Hall effect in magnetic flux pile-up driven antiparallel magnetic field merging and gave analytical solutions of the resistive Hall MHD equations describing stagnation-point ion flows in a thin current sheet-Parker problem. ${ }^{11}$ However, Dorelli's solution for the Hall regime turned out to be basically Parker's solution for the ideal MHD regime. Indeed, the Hall contribution can be transformed away from Dorelli's solution by suitably redefining the velocity gradient associated with the stagnationpoint ion flow. A more complete formulation of the Parker problem in Hall MHD is therefore in order-this is the objective of this paper.

\section{GOVERNING EQUATION FOR HALL MHD}

Consider an incompressible, two-fluid, quasineutral plasma. The governing equations for this plasma dynamics are (in usual notation)

\footnotetext{
${ }^{a)}$ Permanent address: University of Central Florida, Orlando, FL 328161364.
}

$$
\begin{aligned}
& n m_{e}\left[\frac{\partial \mathbf{v}_{e}}{\partial t}+\left(\mathbf{v}_{e} \cdot \nabla\right) \mathbf{v}_{e}\right]=-\nabla p_{e}-n e\left(\mathbf{E}+\frac{1}{c} \mathbf{v}_{e} \times \mathbf{B}\right) \\
&+n e \eta \mathbf{J}, \\
& n m_{i}\left[\frac{\partial \mathbf{v}_{i}}{\partial t}+\left(\mathbf{v}_{i} \cdot \nabla\right) \mathbf{v}_{i}\right]=-\nabla p_{i}+n e\left(\mathbf{E}+\frac{1}{c} \mathbf{v}_{i} \times \mathbf{B}\right) \\
&-n e \eta \mathbf{J}, \\
& \nabla \cdot \mathbf{v}_{e}=0, \\
& \nabla \cdot \mathbf{v}_{i}=0, 0, \\
& \nabla \cdot \mathbf{B}=0,=\frac{1}{c} \mathbf{J}, \\
& \nabla \times \mathbf{B}= \\
& \nabla \times \mathbf{E}=-\frac{1}{c} \frac{\partial \mathbf{B}}{\partial t},
\end{aligned}
$$

where

$$
\mathbf{J} \equiv n e\left(\mathbf{v}_{i}-\mathbf{v}_{e}\right) .
$$

Neglecting electron inertia $\left(m_{e} \rightarrow 0\right)$, Eqs. (1) and (2) can be combined to give a modified ion equation of motion,

$$
n m_{i}\left[\frac{\partial \mathbf{v}_{i}}{\partial t}+\left(\mathbf{v}_{i} \cdot \nabla\right) \mathbf{v}_{i}\right]=-\nabla\left(p_{i}+p_{e}\right)+\frac{1}{c} \mathbf{J} \times \mathbf{B},
$$

and a generalized Ohm's law,

$$
\mathbf{E}+\frac{1}{c} \mathbf{v}_{i} \times \mathbf{B}=\eta \mathbf{J}+\frac{1}{n e c} \mathbf{J} \times \mathbf{B} .
$$

Nondimensionalize distance with respect to a typical length scale $a$, magnetic field with respect to a typical magnetic field strength $B_{0}$, time with respect to the reference Alfvén time $\tau_{A} \equiv a / V_{A_{0}}$, where $V_{A_{0}} \equiv B_{0} / \sqrt{\rho}$ and $\rho \equiv m_{i} n$, and introduce the magnetic and velocity stream functions according to 


$$
\begin{aligned}
& \mathbf{B}=\nabla \psi \times \hat{\hat{\mathbf{i}}_{z}}+b \hat{\hat{\mathbf{i}}_{z}} \\
& \mathbf{v}_{i}=\nabla \phi \times \hat{\mathbf{i}}_{z}+w \hat{\mathbf{i}_{z}}
\end{aligned}
$$

and assume that the physical quantities of interest have no variation along the $z$-direction. The Hall magnetic field $b$ is believed to be produced by the dragging of the in-plane magnetic field in the out-of-plane direction by the magnetized electrons near the magnetic neutral surface. ${ }^{12,13}$ Equations (9) and (10) then yield

$$
\begin{aligned}
& \frac{\partial \psi}{\partial t}+[\psi, \phi]+\sigma[b, \psi]=\hat{\eta} \nabla^{2} \psi, \\
& \frac{\partial b}{\partial t}+[b, \phi]+\sigma\left[\psi, \nabla^{2} \psi\right]+[\psi, w]=\hat{\eta} \nabla^{2} b, \\
& \frac{\partial w}{\partial t}+[w, \phi]=[b, \psi],
\end{aligned}
$$

where

$$
[A, B] \equiv \nabla A \times \nabla B \cdot \hat{\mathbf{i}_{z}}, \quad \sigma \equiv d_{i} / a, \quad \hat{\eta} \equiv \eta c^{2} \tau_{A} / a^{2} .
$$

\section{PARKER PROBLEM IN HALL MHD}

Consider a stagnation-point ion flow at a current sheet separating plasmas of opposite magnetizations ${ }^{11}$ in Hall MHD, governed by Eqs. (12)-(14). Let us assume that the magnetic field lines are straight and parallel to the current sheet. Here, pure resistive annihilation without reconnection of antiparallel magnetic fields (in the $x, y$-plane) occurs. Specifically, consider a unidirectional magnetic field

$$
\mathbf{B}=B_{y}(x) \hat{\mathbf{i}_{y}},
$$

with the boundary condition

$$
B_{y}(0)=0 \text {, }
$$

which is carried toward a neutral sheet at $x=0$ by a stagnation-point ion flow,

$$
\mathbf{v}_{i}=-a x \hat{\hat{\mathbf{i}}_{x}}+a y \hat{\mathbf{i}}_{y}+w \hat{\hat{\mathbf{i}}_{z}} .
$$

Noting that the process in question is steady and that the magnetic field is prescribed as in Eq. (15), Eqs. (12)-(14) become

$$
\begin{aligned}
& E+\frac{\partial \psi}{\partial x} \frac{\partial \phi}{\partial y}-\sigma \frac{\partial b}{\partial y} \frac{\partial \psi}{\partial x}=\hat{\eta} \frac{\partial^{2} \psi}{\partial x^{2}}, \\
& \frac{\partial b}{\partial x} \frac{\partial \phi}{\partial y}-\frac{\partial b}{\partial y} \frac{\partial \phi}{\partial x}+\frac{\partial \psi}{\partial x} \frac{\partial w}{\partial y}=\hat{\eta} \nabla^{2} b, \\
& \frac{\partial w}{\partial x} \frac{\partial \phi}{\partial y}-\frac{\partial w}{\partial y} \frac{\partial \phi}{\partial x}+\frac{\partial \psi}{\partial x} \frac{\partial b}{\partial y}=0,
\end{aligned}
$$

where

$$
E \equiv \frac{\partial \psi}{\partial t}
$$

Dorelli ${ }^{10}$ looked for a solution of Eqs. (18)-(20) of the form

$$
\begin{aligned}
& b=y f(x), \\
& w=\alpha g_{1}(x),
\end{aligned}
$$

where $\alpha$ is a constant. However, this solution restricts the role played by the Hall effect in the process in question. Indeed, the Hall contribution can be transformed away from Dorelli's solution by suitably redefining the velocity gradient $a$ associated with the stagnation-point ion flow (see below). One way to remedy this situation is to recognize the presence of the poloidal shear in the toroidal ion flow, which is intrinsic to the Hall effect. This aspect is also recognized in the Hall resistive tearing mode formulation. ${ }^{14}$ The presence of poloidal shear in the toroidal ion flow basically signifies the generic variation in the out-of-plane ion flow velocity along the outflow direction. On the other hand, the physical mechanism proposed for the generation of the Hall magnetic field $b$ (Refs. 12 and 13) involves a poloidal shear in the toroidal electron flow, some of which appears to be coupled also to the toroidal ion flow. Therefore, we incorporate a poloidal shear into the toroidal ion flow according to

$$
w=\alpha g_{1}(x)+\frac{\beta}{2} y^{2} g_{2}(x),
$$

where $\beta$ is a constant characterizing this poloidal shear. The particular form of poloidal shear superposed on the toroidal ion flow used in Eq. (22) is motivated by the symmetry properties of Eqs. (12)-(14) in the ideal limit, which indicate $\psi$ and $w$ to be even functions of both $x$ and $y$, and $\phi$ and $b$ to be odd function of both $x$ and $y ;{ }^{15}$ these properties are sustained by the solutions in the following. Using Eqs. (21a) and (22), Eq. (20) gives

$$
\left(\alpha g_{1}^{\prime}+\frac{\beta}{2} y^{2} g_{2}^{\prime}\right)(-a x)-\left(\beta y g_{2}\right)(-a y)+\frac{\partial \psi}{\partial x} f^{\prime}=0,
$$

where primes denote differentiation with respect to $x$. We obtain from Eq. (23)

$$
\begin{aligned}
& (-a x)\left(\alpha g_{1}^{\prime}\right)=-\frac{\partial \psi}{\partial x} f^{\prime}, \\
& g_{2}(x)=x^{2} .
\end{aligned}
$$

Using Eqs. (21a), (22), (24), and (25), Eq. (19) then gives

$$
y f^{\prime}(-a x)-f(-a y)+\frac{\partial \psi}{\partial x}\left(\beta y x^{2}\right)=\hat{\eta} y f^{\prime \prime},
$$

or

$$
f^{\prime \prime}+\frac{a}{\hat{\eta}}\left(x f^{\prime}-f\right)=\frac{\beta}{\hat{\eta}} x^{2} \frac{\partial \psi}{\partial x} .
$$

Recognizing that the Hall effect becomes important away from the current sheet at $x=0$ (in what was called the "intermediate" layer by Terasawa ${ }^{16}$ in his investigation of the Hall resistive tearing mode), a reasonable approximate solution of Eq. (27) is 


$$
f(x) \approx A x-\frac{\beta}{a} x^{2} \frac{\partial \psi}{\partial x},
$$

where $A$ is a constant.

Using Eqs. (21a) and (28), Eq. (18) gives

$$
-E+\frac{\partial \psi}{\partial x}(-a x)-\sigma\left(A x-\frac{\beta}{a} x^{2} \frac{\partial \psi}{\partial x}\right) \frac{\partial \psi}{\partial x}=\hat{\eta} \frac{\partial^{2} \psi}{\partial x^{2}},
$$

or

$$
E=\hat{\eta} B_{y}^{\prime}+(a+\sigma A) x B_{y}+\sigma \frac{\beta}{a} x^{2} B_{y}^{2},
$$

which is a generalization of Parker's equation to Hall MHD. Observe that, for Dorelli's solution (which corresponds to $\beta=0$ ), the Hall contribution (represented by the term corresponding to $\sigma$ ) can be simply transformed away by redefining the velocity gradient $a$ associated with the stagnationpoint ion flow.

It is important to note that Eq. (29) shows that the tripledeck structure (borrowing the terminology from boundary layer theory in fluid dynamics ${ }^{17}$ ) in Hall resistive MHD (originally pointed out by Terasawa ${ }^{16}$ ) is operational for the Parker problem in Hall MHD, as to be expected. Thus, we have

(i) a resistive region near the current sheet at $x=0$,

(ii) an ideal MHD region away from the current sheet at $x=0$, and

(iii) a Hall resistive region in between (i) and (ii)—called the intermediate region by Terasawa. ${ }^{16}$

In the resistive region, Eq. (29) may be approximated by

$$
E \approx \hat{\eta} B_{y}^{\prime},
$$

which gives Parker's solution,

$$
B_{y} \approx \frac{E}{\hat{\eta}} x .
$$
by

In the ideal MHD region, Eq. (29) may be approximated

$$
E \approx(a+\sigma A) x B_{y}+\sigma \frac{\beta}{a} x^{2} B_{y}^{2},
$$

which gives modified Dorelli's solution,

$$
B_{y} \approx\left[\frac{\sigma \beta}{2 a(a+\sigma A)}\right]\left[-1+\sqrt{1+\frac{4 E(a+\sigma A) a^{2}}{\sigma^{2} \beta^{2}}}\right] \frac{1}{x} .
$$

In the Hall resistive region, Eq. (29) may be approximated by

$$
E \approx \hat{\eta} B_{y}^{\prime}+\sigma \frac{\beta}{a} x^{2} B_{y}^{2},
$$

which gives

$$
B_{y} \approx\left(\frac{3 \hat{\eta} a}{\sigma \beta}\right) \frac{1}{x^{3}} .
$$

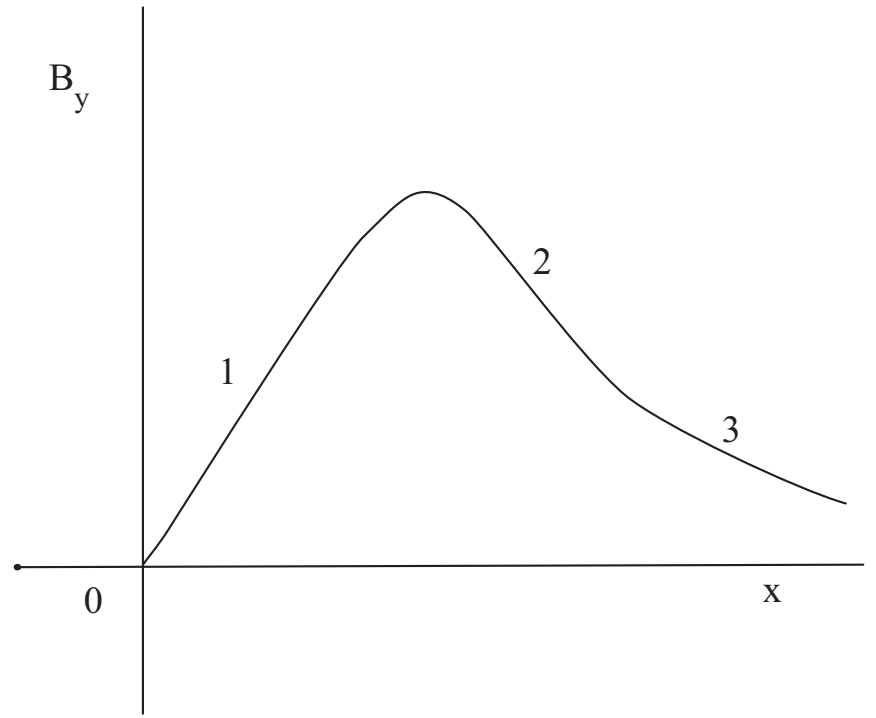

FIG. 1. Magnetic field profile for the Parker problem in Hall MHD. (1) $B_{y} \sim x$, (2) $B_{y} \sim 1 / x^{3}$, and (3) $B_{y} \sim 1 / x$

The triple-deck structure given by Eqs. (31), (33), and (35) is shown in Fig. 1. Noting from Eqs. (21a) and (28) that

$$
b=y\left(A x+\frac{\beta}{a} x^{2} B_{y}\right),
$$

one observes that both in the

- Hall resistive region, $B_{y} \sim 1 / x^{3}$, and

- ideal MHD region, $B_{y} \sim 1 / x$,

$b$ has a quadrupolar structure. So, Hall effects materialize only via their signature - the quadrupolar out-of-plane magnetic field pattern. ${ }^{18}$

\section{DISCUSSION}

In recognition of the fact that a signature of the Hall effect is the generation of out-of-plane "separator" components of the magnetic and ion-flow velocity fields, a more accurate representation of the latter appears to be in order for a more complete formulation of the Parker problem. In this paper, this is accomplished by incorporating poloidal shear into the toroidal ion flow associated with the Hall effect. This is found to lead to a triple-deck structure for the Parker problem in Hall MHD, in accordance with the idea originally put forward by Terasawa. ${ }^{16}$ The magnetic field is found to fall off in the intermediate Hall-resistive region more steeply (like $\left.1 / x^{3}\right)$ than that (like $1 / x$ ) in the outer ideal MHD region.

\section{ACKNOWLEDGMENTS}

I acknowledge with gratitude the stimulating interactions with Dr. Luis Chacon that led to this work. I am thankful to the referee for his helpful remarks. My thanks are also due to Dr. Michael Shay and Dr. Michael Johnson for helpful communications and discussions. 
${ }^{1}$ D. Biskamp, Phys. Fluids 29, 1520 (1986).

${ }^{2}$ P. A. Sweet, in Electromagnetic Phenomena in Cosmic Physics, edited by

B. Lehnert (Cambridge University Press, Cambridge, 1958), p. 123.

${ }^{3}$ E. N. Parker, J. Geophys. Res. 62, 509, DOI: 10.1029/JZ062i004p00509 (1957).

${ }^{4}$ In the Sweet-Parker model, the bottleneck in the outflow is produced by slowly moving ions under the influence of a very small transverse magnetic field in the elongated diffusion region.

${ }^{5}$ D. A. Knoll and L. Chacon, Phys. Plasmas 13, 032307 (2006).

${ }^{6}$ A. Clark, Phys. Fluids 7, 1299 (1964).

${ }^{7}$ B. U. O. Sonnerup, in Solar System Plasma Physics, edited by L. J. Lanzerotti, C. F. Kennel, and E. N. Parker (North Holland, Amsterdam, 1979), p. 45 .

${ }^{8}$ J. C. Dorelli and J. Birn, J. Geophys. Res. 108, 1133, DOI: 10.1029/ 2001JA009180 (2003).

${ }^{9}$ D. A. Knoll and L. Chacon, Phys. Rev. Lett. 96, 135001 (2006).
${ }^{10}$ J. C. Dorelli, Phys. Plasmas 10, 3309 (2003).

${ }^{11}$ E. N. Parker, J. Plasma Phys. 9, 49 (1973).

${ }^{12}$ J. F. Drake, M. A. Shay, and M. Swisdak, Phys. Plasmas 15, 042306 (2008).

${ }^{13}$ M. Yamada, Phys. Plasmas 14, 058102 (2007)

${ }^{14}$ B. K. Shivamoggi, Europhys. Lett. 83, 55002 (2008).

${ }^{15}$ B. K. Shivamoggi, Europhys. Lett. 85, 25001 (2009).

${ }^{16}$ T. Terasawa, Geophys. Res. Lett. 10, 475, DOI: 10.1029/ GL010i006p00475 (1983).

${ }^{17}$ F. T. Smith, IMA J. Appl. Math. 28, 207 (1982).

${ }^{18}$ Laboratory experiments (Refs. 19 and 20) confirmed this signature of the Hall effect.

${ }^{19}$ Y. Ren, M. Yamada, S. Gerhardt, H. Ji, R. Kulsrud, and A. Kuritsyn, Phys. Rev. Lett. 95, 055003 (2005).

${ }^{20}$ A. Frank, S. G. Bugrov, and V. S. Markov, Phys. Plasmas 15, 092102 (2008). 